\title{
High expression of TMEM40 contributes to progressive features of tongue squamous cell carcinoma
}

\author{
QINGYAN ZHANG ${ }^{1}$, DANHUI HUANG $^{1}$, ZHENFEI ZHANG $^{2}$, YUZHEN FENG $^{2}$, MEITING FU $^{1}$, \\ MIN WEI $^{2}$, JUEYU ZHOU ${ }^{2}$, YUANJIN HUANG ${ }^{3}$, SHUGUANG LIU ${ }^{3}$ and RONG SHI ${ }^{2}$ \\ ${ }^{1}$ The First School of Clinical Medicine, Southern Medical University; \\ ${ }^{2}$ Department of Biochemistry and Molecular Biology, School of Basic Medical Sciences, \\ Southern Medical University, Guangzhou, Guangdong 510515; ${ }^{3}$ Stomatological Hospital, \\ Southern Medical University, Guangzhou, Guangdong 510280, P.R. China
}

Received March 18, 2018; Accepted September 10, 2018

DOI: $10.3892 /$ or.2018.6788

\begin{abstract}
Transmembrane protein 40 (TMEM40) is a 23-kDa protein and its association with tongue squamous cell carcinoma (TSCC) remains unclear. This study aimed to investigate the expression and clinical significance of TMEM40 in TSCC and its roles in TSCC cells. Immunohistochemical analysis was performed to detect the expression levels of TMEM40 in 60 tongue tissue samples. Furthermore, TMEM40 was overexpressed and inhibited in two TSCC cell lines by transfection with pEZ-M98-TMEM40 plasmid or TMEM40 small interfering RNA, respectively. Cell Counting Kit- 8 and colony formation assays were used to investigate the effects of TMEM40 on cell proliferation and colony formation ability, respectively. Flow cytometry was performed to determine cell apoptosis and cycle conditions of transfected cells. Wound-healing and
\end{abstract}

Correspondence to: Professor Rong Shi, Department of Biochemistry and Molecular Biology, School of Basic Medical Sciences, Southern Medical University, 1023 Sha Tai Nan Road, Guangzhou, Guangdong 510515, P.R. China

E-mail: shirongphd@126.com

Professor Shuguang Liu, Stomatological Hospital, Southern Medical University, 366 Jiangnan Boulevard South, Guangzhou, Guangdong 510280, P.R. China

E-mail: ilsg00@126.com

Abbreviations: TSCC, tongue squamous cell carcinoma; TMEM40, transmembrane protein 40; pTNM, pathological TNM; H\&E, hematoxylin-eosin; IHC, immuohistochemistry; TMA, tissue microarray; DMEM/F12, Dulbecco's Modified Eagle's medium/Nutrient Mixture F-12; FBS, fetal bovine serum; NC, negative control; qRT-PCR, quantitative real-time PCR; PVDF, polyvinylidene fluoride; CCK-8, Cell Counting Kit-8; ROC, receiver operating characteristic; siRNA, small interfering RNA; Bax, Bcl-2 associated X protein; MMP-9, matrix metallopeptidase 9

Key words: TMEM40, tongue squamous cell carcinoma, immunohistochemistry, clinicopathological parameters, proliferation, invasion
Transwell assays were processed to explore the effects of TMEM40 on cell migration and invasion, respectively. The results indicated that TMEM40 expression levels were significantly increased in TSCC tissues compared with adjacent normal tongue tissues $(\mathrm{P}<0.01)$. Clinicopathological analysis revealed that TMEM40 expression was positively correlated with pathological TNM (pTNM) status $(\mathrm{P}<0.05)$, histological grade $(\mathrm{P}<0.001)$ and clinical stage $(\mathrm{P}<0.01)$, but not with sex or age. Results of cell proliferation, apoptosis, migration and invasion assays indicated that when TMEM40 had been successfully overexpressed or knocked down in CAL27 and SCC9 TSCC cell lines, cell growth and invasion increased in the TMEM40 overexpressing cells, while they decreased in TMEM40-knockdown cells. Furthermore, experiments revealed that TMEM40 knockdown resulted in increased levels of p53 and Bax, and decreased levels of MMP-9, which indicated that TMEM40 regulated cell apoptosis and migration via involvement of p53, Bax and MMP-9 in TSCC cells. Our findings indicated that increased expression of TMEM40 contributed to progressive features of TSCC via regulation of p53, Bax and MMP-9.

\section{Introduction}

Tongue squamous cell carcinoma (TSCC) is the most prevalent malignancy of the head and neck and accounts for $~ 90 \%$ of oropharyngeal and oral malignancies (1). Over 300,000 new cases and over 100,000 deaths from oral cavity cancer are estimated to occur worldwide every year (2). Epidemiological studies revealed that smoking, alcohol use, smokeless tobacco use and HPV infection are important risk factors for TSCC (3). Increasingly, it is acknowledged that TSCC arises from genetic and epigenetic mutations (4). TSCC therapy mainly focuses on surgery, combined with radio- or chemotherapy. The 5-year survival rate for patients with TSCC is $\sim 60 \%$ after arduous treatment (5). However, traditional therapies can cause an impact on the functions of surrounding organs, leading to eating, drinking, chewing and swallowing difficulties (6). Recently, targeted cancer therapy has developed rapidly. Epidermal growth factor receptor inhibitors have shown initial success in recurrent and metastatic head and neck squamous 
cell carcinoma and treatments include monoclonal antibodies, which proves the necessity of identifying and defining diagnostic and prognostic biomarkers (7).

The transmembrane protein 40 (TMEM40) gene encodes a protein of 233 amino acids and is located on chromosome 3p25.2. Little is known about the functions of TMEM40. A study has indicated that TMEM40 expression is associated with the damage of the parietal lobule (8). A previous study has suggested that TMEM40 is overexpressed in bladder cancer and described the association between TMEM40 and clinical parameters of bladder cancer tissues. The present study aims to assess the functions of TMEM40 in TSCC. We demonstrated that TMEM40 was upregulated in tumor cells of clinical TSCC tissue samples and we evaluated the association of TMEM40 with clinical characteristics. Moreover, to explore the functions of TMEM40 in TSCC cells, we evaluated the effects of TMEM40 overexpression and TMEM40 knockdown on the progression of TSCC cells in vitro. These results indicated that TMEM40 served an important role in TSCC cell proliferation and migration and represented a potential oncogene.

\section{Materials and methods}

Tissue collection. A total of 50 TSCC and 10 cancer-adjacent normal tongue tissues were collected at the Stomatology Hospital of Southern Medical University (China) between January 2013 and December 2015. Patients did not receive any treatment and had no history of other malignancies prior to tumor surgery. Recorded clinicopathological parameters included sex, age, pTNM status, histology grade and clinical stage. All experimental procedures were approved by the Ethics Committee of Southern Medical University. All patients provided written informed consent.

Immunohistochemical analysis. All tissue samples were examined for TMEM40 expression by immunohistochemistry (IHC) using hematoxylin-eosin (H\&E) stain and a phase-contrast microscope (Nikon Eclipse Ti-S; Nikon Corp., Tokyo, Japan). Firstly, a tissue microarray (TMA) was constructed according to a standard method. Then, the TMA block was cut into 5- $\mu \mathrm{m}$ sections for IHC analysis. It was processed based on standard procedures (9). Tissues were dehydrated and incubated with $5 \%$ normal goat serum for blocking. Subsequently, tissues were incubated with TMEM40 primary antibody (mouse monoclonal; dilution 1:200; cat. no. sc-393601; Santa Cruz Biotechnology, Inc., Santa Cruz, CA, USA) at $4^{\circ} \mathrm{C}$ overnight followed by incubation with goat anti-rabbit secondary antibody [dilution 1:5,000; cat. no. 70-GAR007; Hangzhou MultiSciences (Lianke) Biotech Co., Ltd., Hangzhou, China]. Sections were visualized using a DAB Horseradish Peroxidase (HRP) Color Development kit (Beyotime Institute of Biotechnology, Haimen, China) and counterstained with hematoxylin. Two independent investigators determined the staining score by multiplying the staining intensity and the percentage of positive cells. A receiver operating characteristic (ROC) curve analysis, based on clinicopathological parameters, was used to obtain the optimal cut-off score. If the TMEM40 score was below the cut-off score, tissue was defined as TMEM40 negative; otherwise it was TMEM40 positive.
Cell culture. Human tongue cell lines CAL27 and SCC9 were purchased from the China Center for Typical Culture Collection (CCTCC; Wuhan, China). Cells were cultured in Dulbecco's modified Eagle's medium/nutrient mixture F-12 (DMEM/F12; Gibco; Thermo Fisher Scientific, Inc., Waltham, MA, USA) supplemented with $10 \%$ fetal bovine serum (FBS; HyClone Laboratories; GE Healthcare Life Sciences, Logan, UT, USA) and $100 \mathrm{U} / \mathrm{ml}$ penicillin/streptomycin and cells were maintained at $37^{\circ} \mathrm{C}$ in a humidified atmosphere with $5 \% \mathrm{CO}_{2}$.

Plasmid DNA and siRNA transfection. Plasmid DNA was transfected to evaluate TMEM40 overexpression. The TMEM40 sequence and negative control were synthesized and cloned into a pEZ-M98 vector (Guangzhou Fulengen Co., Ltd., Guangzhou, China). According to the manufacturer's protocol, the pEZ-M98-TMEM40 plasmid or empty pEZ-M98 vector were used to transfect CAL27 and SCC9 cells using Lipofectamine 2000 transfection reagent (Invitrogen; Thermo Fisher Scientific, Inc.) and cultured in 6-well plates. siRNAs were used to knockdown TMEM40 expression. siRNAs targeting TMEM40 and a negative control (NC) were purchased from Guangzhou RiboBio Co., Ltd. (Guangzhou, China). The specific siRNA sequences were as follows: TMEM40-siRNA-1, 5'-GGATGAGCTTCAACT CTAT-3'; TMEM40-siRNA-2, 5'-GTGGAGGCCTCTCAG TTAA-3'; NC, 5'-UUCUCCGAACGUGUCACGUTT-3'. Total RNAs were isolated $48 \mathrm{~h}$ after transfection to detect TMEM40 mRNA expression levels.

RNA isolation and reverse transcription-quantitative polymerase chain reaction ( $R T-q P C R$ ). To quantify TMEM40 mRNA expression, total RNAs were isolated from the cells $48 \mathrm{~h}$ after transfection. Total RNAs were extracted using RNAiso Plus (Takara Biotechnology Co., Ltd., Dalian, China) according to the manufacturer's protocol and dissolved into nuclease-free water. A PrimeScript ${ }^{\circledR}$ RT reagent kit (Takara Biotechnology Co., Ltd., Dalian, China) was used for RT of mRNA to cDNA. Then, qPCR was performed to detect TMEM40 expression levels using the SYBR ${ }^{\circledR}$ Premix Ex TaqTM II kit (Takara Biotechnology Co., Ltd.) and an ABI 7500 real-time PCR amplifier (Applied Biosystems; Thermo Fisher Scientific, Inc.). The thermal cycling conditions were as follows: $95^{\circ} \mathrm{C}$ for $10 \mathrm{~min}$, followed by 40 cycles at $95^{\circ} \mathrm{C}$ for $15 \mathrm{sec}$ and $60^{\circ} \mathrm{C}$ for $34 \mathrm{sec}$. The following primers were used: TMEM40 forward, 5'-CAGAGCAACCGGAAAACATCG-3' and reverse, 5'-CTGGGCTACACTGAGCACC-3'; GAPDH forward, 5'-AGAAGGCTGGGGCTCATTTG-3' and reverse, 5'-AAGTGGTCGTTGAGGGCAATG-3'. Each reaction was analyzed in triplicate. The comparative $\mathrm{Ct}\left(2^{-\Delta \Delta \mathrm{Cq}}\right)(10)$ method was used to calculate the relative levels of TMEM40.

Western blotting. To detect TMEM40 protein expression, total proteins were extracted from cells $72 \mathrm{~h}$ after transfection. Radioimmunoprecipitation assay buffer containing $1 \mathrm{mM}$ PMSF (Beyotime Institute of Biotechnology) was used. Protein concentrations were determined using a BCA Protein Assay kit (Beyotime Institute of Biotechnology). Protein samples were mixed with loading buffer [4:1 (v/v); Beyotime Institute of Biotechnology] and were boiled for $10 \mathrm{~min}$. 
Table I. Association of TMEM40 expression with normal tongue and TSCC tissues.

TMEM40 staining

\begin{tabular}{lcccr} 
& All cases $(\%)$ & Negative expression $(\%)$ & Positive expression (\%) & P-value \\
\hline Squamous cell carcinoma & 50 & $28(56.0)$ & $22(44.0)$ & 0.008 \\
Normal tongue tissues & 10 & $10(100.0)$ & $0(0.0)$ & \\
\hline
\end{tabular}

aP-values were obtained using Chi-square test. TMEM40, transmembrane protein 40; TSCC, tongue squamous cell carcinoma.

Samples were separated by $10 \%$ SDS-PAGE and transferred to a polyvinylidene fluoride membrane (EMD Millipore, Billerica, MA, USA) with a wet-transfer machine (Trans-blot SD; Bio-Rad Laboratories, Inc., Hercules, CA, USA). The membrane was blocked with $5 \%$ non-fat milk at room temperature for $2 \mathrm{~h}$ and incubated with TMEM40 primary antibody (mouse monoclonal; 1:500; Santa Cruz Biotechnology, Inc.) and $\beta$-actin primary antibody (mouse monoclonal; $1: 2,000$; ProteinTech Group, Inc., Chicago, IL, USA) at $4^{\circ} \mathrm{C}$ overnight. The membrane was washed with $1 \mathrm{X}$ TBST (3X) and incubated with HRP-conjugated secondary antibody (goat anti-mouse IgG; 1:5,000; NeoBioscience, Shenzhen, China) at room temperature for $2 \mathrm{~h}$, followed by washing with $1 \mathrm{X}$ TBST (3x). Immunoreactivebandswere visualized withtheSmartChemi610 (Beijing Sage Creation Science Co., Ltd., Beijing, China) using the Immobilon Western Chemiluminescent HRP Substrate (EMD Millipore).

Cell viability assay. Cell Counting Kit-8 (CCK-8; Nanjing KeyGen Biotech Co., Ltd., Nanjing, China) was used to assess cell viability. A total of 1,000-2,000 transfected cells were seeded in 96-well plates and incubated for 3 days. At the indicated time-point, $10 \mu \mathrm{l}$ of the CCK- 8 stock solution was added to each well together with $100 \mu 1$ medium and incubated at $37^{\circ} \mathrm{C}$ for $2 \mathrm{~h}$. The absorbance value (optical density) was measured at $450 \mathrm{~nm}$ using a multifunctional microplate reader (Tecan Infinite M200; Tecan Group Ltd., Mannedorf, Switzerland). All experiments were performed in triplicate and five duplicate wells were screened.

Colony formation assay. A total of $2 \mathrm{ml}$ complete medium containing 2,000 transfected cells was added to 6-well plates and incubated at $37^{\circ} \mathrm{C}$ with $5 \% \mathrm{CO}_{2}$ for 10 days. After discarding the supernatant, cells were washed with phosphatebuffered saline (PBS) (3X), fixed using $500 \mu 1$ of methanol at room temperature for $20 \mathrm{~min}$ and stained with $0.1 \%$ crystal violet (Nanjing KeyGen Biotech Co., Ltd.) for $20 \mathrm{~min}$. The colony formation efficacy was determined. Assays were performed in triplicate.

Evaluation of cell apoptosis and cell cycle distribution by flow cytometry. Flow cytometry was used to evaluate the cell apoptosis ratio of the transfected cells. Harvested cells were double-labeled with Annexin V-FITC and PI apoptosis detection kits (Nanjing KeyGen Biotech Co., Ltd.) and a BD FACSVerse flow cytometer (BD Biosciences, Franklin Lakes, NJ, USA) was used for the detection of apoptosis. For cell cycle measurements, transfected cells were harvested, digested with $0.05 \%$ trypsin and fixed with $70 \%$ ethanol at $-20^{\circ} \mathrm{C}$ overnight. Cells were stained with $0.1 \mathrm{mg} / \mathrm{ml}$ propidium iodide (PI; Sigma-Aldrich; Merck KGaA, Darmstadt, USA) for $30 \mathrm{~min}$. A FACSVerse flow cytometer was used to measure the intensities of the fluorescence signals. A minimum of 20,000 cells was observed for each sample. Percentages of cells in the G0/G1, S and G2/M phases were evaluated using FlowJo software (Tree Star, Inc., Ashland, OR, USA). All experiments were performed in triplicate.

Wound-healing assay. A wound-healing assay was performed to assess cell migration. When cells reached a density of $80-90 \%$ after transfection, a $10-\mu 1$ pipette tip was used to inflict a straight wound. PBS was used to rinse the cell debris from the dishes and serum free medium was added for cell culturing. Scrape lines were based on the border of the majority of migratory cells and photographed using a phase-contrast microscope (Nikon Eclipse Ti-S; Nikon Corp.) at different time-points $(0,12,24$ and $48 \mathrm{~h})$. The scratch area and wound-healing percentage were calculated using ImageJ software (National Institutes of Health, Bethesda, MD, USA). The experiment was repeated in triplicate and we selected the simultaneous groups.

Cell invasion assay. Cell invasion assays were performed using Transwell chambers $(8-\mu \mathrm{m}$ pore size; Corning Inc., Corning, NY, USA) in 24-well plates. Transwell chambers were coated with $30 \mu 1$ Matrigel (Corning Inc.) and incubated for $60 \mathrm{~min}$ at $37^{\circ} \mathrm{C}$. Transfected CAL27 and SCC9 cells were counted and $1 \times 10^{5}$ cells/chamber were seeded in serum-free DMEM/F12 medium. The lower well was filled with $800 \mu \mathrm{l}$ complete medium. After $36 \mathrm{~h}$ of culturing at $37^{\circ} \mathrm{C}$ in a $5 \% \mathrm{CO}_{2}$ incubator, the cells in the upper layer were removed with a swab. The cells on the bottom of the Transwell chamber were fixed with methanol and stained with $0.1 \%$ crystal violet (Nanjing KeyGen Biotech Co., Ltd.). The results were photographed at five randomized visual fields and experiments were repeated three times.

Statistical analysis. All statistical analyses were completed using the SPSS 20.0 software. Data are presented as the mean \pm SD. ROC was adopted to select an optimal cut-off score for TMEM40 positive. A t-test was used to evaluate differences in expression between two groups. One-way ANOVA followed by the Tukey's post hoc test was used to analyze the CCK-8 assay data. A Chi-square test was used to 
A

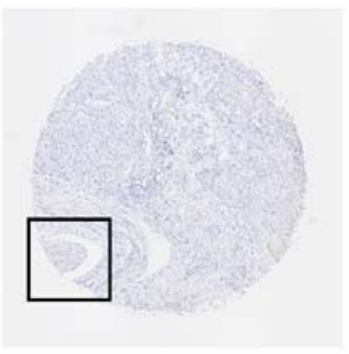

E

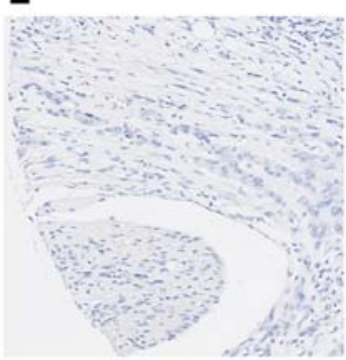

B

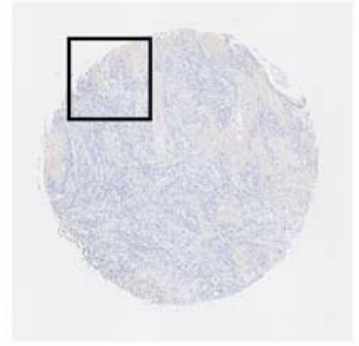

$\mathbf{F}$

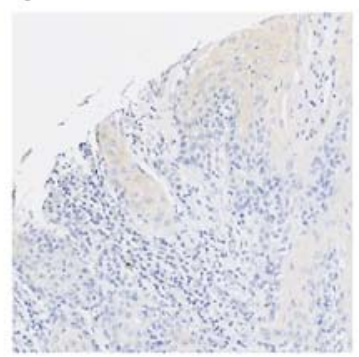

C

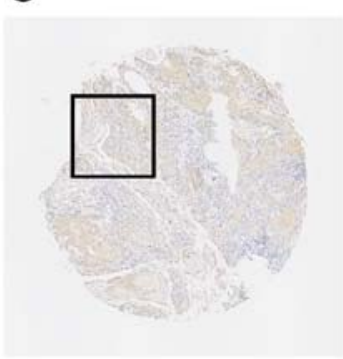

G

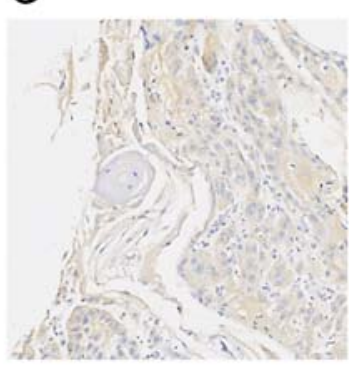

D

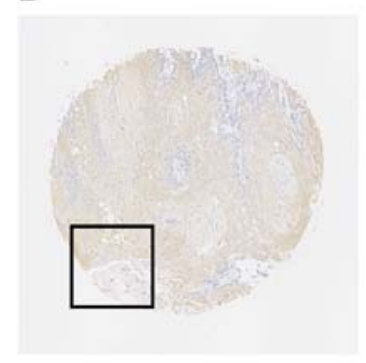

H

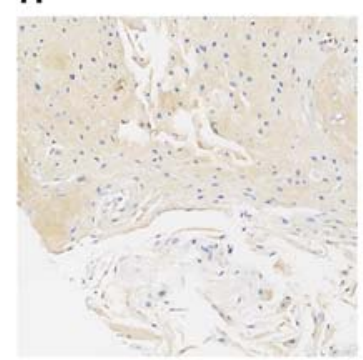

Figure 1. IHC results for the expression of TMEM40 in normal tongue and TSCC tissues. (A) Negative expression of TMEM40 was detected in normal tongue tissue (case 2; magnification, x100). (B) Negative expression of TMEM40 was detected in TSCC tissue (case 28) with 30\% of tumor cells exhibiting positive staining (magnification, x100). (C) Positive expression of TMEM40 was revealed in TSCC tissue (case 36) with 80\% staining extensity and moderate intensity (magnification, x100). (D) Positive expression of TMEM40 was revealed in TSCC tissue (case 44) with 95\% staining extensity and strong intensity (magnification, x100). (E-H) represent higher magnifications (x400) of the areas highlighted with a black box in (A-D), respectively. IHC, immunohistochemistry; TMEM40, transmembrane protein 40; TSCC, tongue squamous cell carcinoma.

assess the association between the IHC staining score and the clinicopathological parameters of TSCC. Differences were considered statistically significant at $\mathrm{P}<0.05$.

\section{Results}

TMEM40 expression in tissue samples of TSCC and its association with clinicopathological parameters. To investigate the protein expression level of TMEM40 in TSCC tissues, IHC was performed in 50 TSCC and 10 cancer-adjacent normal tongue tissue sections, which were formalin-fixed and paraffin-embedded. Four representative samples of different levels of TMEM40 IHC staining are shown in Fig. 1. As shown in Table I, TMEM40 was observed in $44.0 \%$ (22/50) of the TSCC specimens and $0.00 \%(0 / 10)$ of the normal tongue tissues. The results indicated that TMEM40 expression was significantly increased in tumor tissues compared with the normal tongue tissues $\left(\chi^{2}=2.756 ; \mathrm{P}=0.008\right.$; Table I).

The results of the association analysis between clinicopathological characteristics and TMEM40 protein expression levels in 50 patients with TSCC are summarized in Table II. The results indicated that high TMEM40 expression levels were correlated with pTNM status $\left(\chi^{2}=4.884 ; \mathrm{P}=0.027\right)$, histological grade $\left(\chi^{2}=17.949 ; \mathrm{P}<0.001\right)$ and clinical stage $\left(\chi^{2}=10.189 ; \mathrm{P}=0.006\right)$. However, there was no association between TMEM40 expression levels and sex or age.

Specific plasmid vectors and siRNA upregulate and silence TMEM40 expression, respectively. TSCC cell lines CAL27 and SCC9 were cultured and transfected with pEZ-M98-TMEM40 plasmid or empty pEZ-M98 vector, or siRNA or negative control. The results revealed that $40 \%$ of the transfected pEZ-M98-TMEM40 plasmid or empty pEZ-M98 vector cells
Table II. Association of TMEM40 expression and clinicopathological features in TSCC tissues.

TMEM40 staining

\begin{tabular}{lcccc} 
Variables & Negative (\%) & Positive (\%) & Total & P-value $^{\text {b }}$ \\
\hline Sex & & & & 0.755 \\
Male & 24 & 13 & 37 & \\
Female & 14 & 9 & 23 & \\
Age (years) & & & & 0.464 \\
$\leq 53.3^{\text {a }}$ & 21 & 10 & 31 & \\
$>53.3$ & 17 & 12 & 29 & \\
pT Status & & & & 0.027 \\
T1 & $15(75.0)$ & $5(25.0)$ & 20 & \\
T2-T4 & $13(43.3)$ & $17(56.7)$ & 30 & \\
Grade & & & & $<0.001$ \\
1 & $28(71.8)$ & $11(28.2)$ & 39 & \\
2 & $0(0.0)$ & $6(100.0)$ & 6 & \\
3 & $0(0.0)$ & $5(100.0)$ & 5 & \\
Stage & & & & 0.006 \\
I & $16(76.2)$ & $5(23.8)$ & 21 & \\
II & $12(50.0)$ & $12(50.0)$ & 24 & \\
III & $0(0.0)$ & $5(100.0)$ & 5 & \\
\end{tabular}

${ }^{\mathrm{a}}$ Mean age. ${ }^{\mathrm{b}} \mathrm{P}$-values were obtained using Chi-square test. TMEM40, transmembrane protein 40; TSCC, tongue squamous cell carcinoma.

died with the transfection experiment while no cells died with transfection of siRNA or the negative control. The expression 

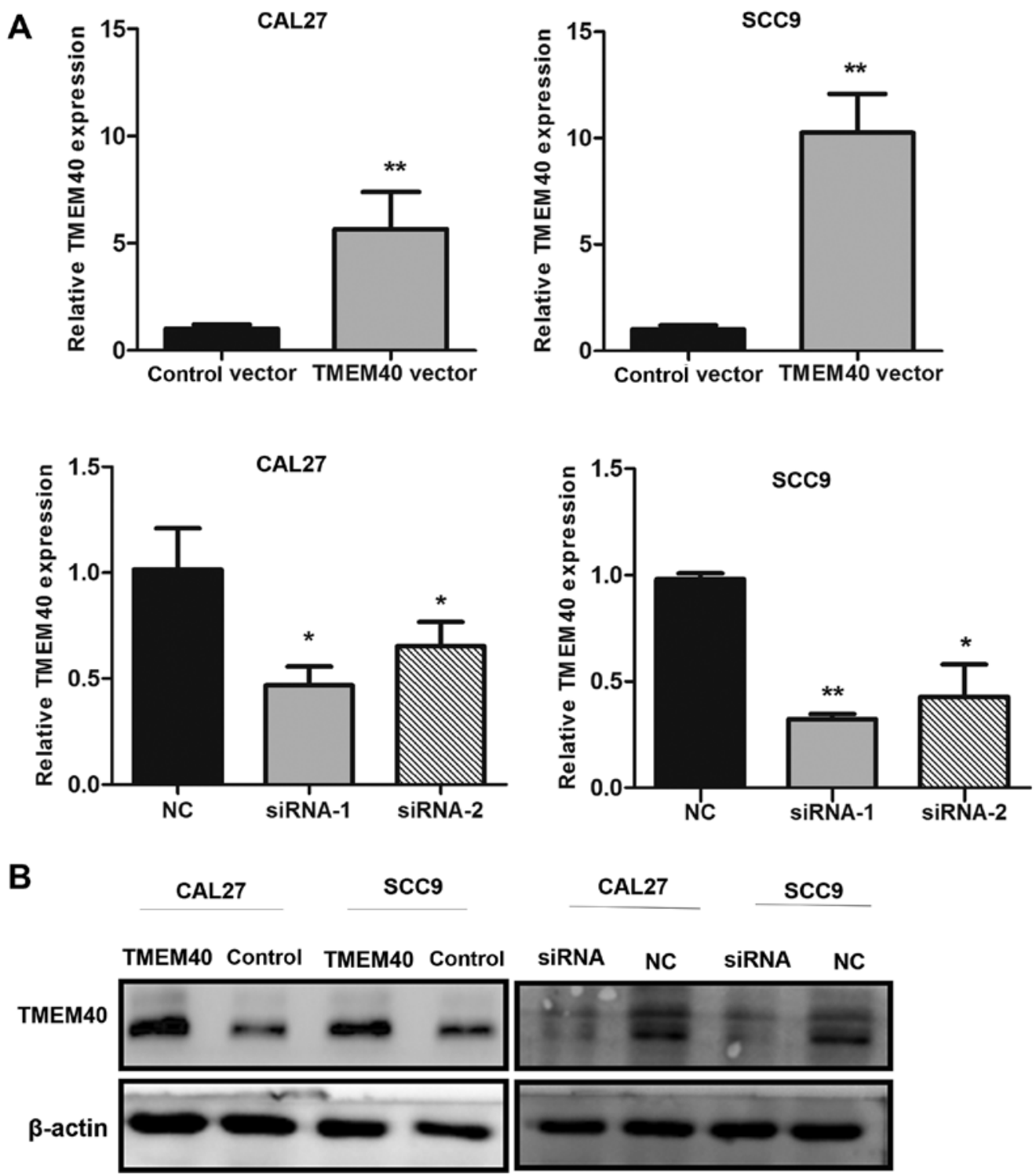

Figure 2. RT-qPCR and western blot results for TMEM40 expression in CAL27 and SCC9 transfected with TMEM40 vector or TMEM40-siRNA compared with controls. (A) RT-qPCR results for CAL27 and SCC9 transfected with control vector, TMEM40 vector, siRNA-1, siRNA-2 and NC. (B) Western blot analysis revealed significant upregulation in transfected TMEM40 vector cells and downregulation in TMEM40-siRNA cells in comparison with the controls. $\beta$-actin was used as internal control. ${ }^{*} \mathrm{P}<0.05,{ }^{* * *} \mathrm{P}<0.01$. RT-qPCR, reverse transcription-quantitative polymerase chain reaction; TMEM40, transmembrane protein $40 ; \mathrm{NC}$, negative control.

level of TMEM40 was analyzed by RT-qPCR and amplification efficiency by qPCR was estimated at $98-100 \%$ for all samples. The results revealed that the relative mRNA expression levels of TMEM40 in plasmid-transfected cells were significantly upregulated and in siRNA-transfected cells were significantly downregulated (Fig. 2A; $\mathrm{P}<0.05 ; \mathrm{P}<0.01$ ). As shown in Fig. 2A, the RT-qPCR results revealed that both TMEM40 siRNAs (TMEM40-siRNA-1 and TMEM40-siRNA-2) efficiently silenced TMEM40 expression in CAL27 and SCC9 cells. TMEM40-siRNA-1, which exhibited a more pronounced inhibitory effect, was selected for the following experiments. Western blot analysis was performed to detect the protein expression levels of TMEM40, which revealed consistent results (Fig. 2B).

Role of TMEM40 in TSCC cell proliferation. To explore the influence of TMEM40 on the growth of TSCC cells, CCK-8 assays were applied to determine the cell viability of CAL27 and SCC9 cells. The pEZ-M98-TMEM40 plasmid-transfected cells increased significantly in cell viability compared with the negative control group (Fig. 3A). In contrast, TMEM40-siRNA suppressed the growth of CAL27 and SCC9 cells (Fig. 3A). The results indicated that TMEM40 induced cell proliferation in TSCC cells.

Effects of TMEM40 on colony formation abilities of TSCC cells. The role of TMEM40 in the regulation of colony formation in TSCC cells was investigated. CAL27 and SCC9 cells transfected with pEZ-M98-TMEM40 plasmid indicated a significant higher colony-forming efficacy compared with the control group, which was transfected with empty pEZ-M98-TMEM40 plasmid. Low expression of TMEM40 suppressed colony formation abilities of cells transfected with TMEM40-siRNA (Fig. 3B and C; $\mathrm{P}<0.05 ; \mathrm{P}<0.01 ; \mathrm{P}<0.001$ ). 
A
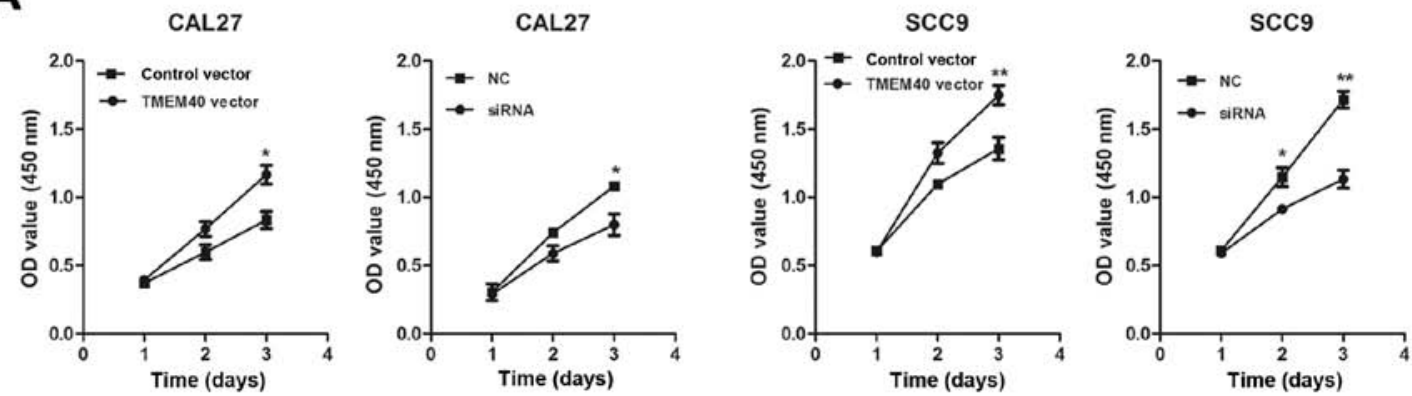

B

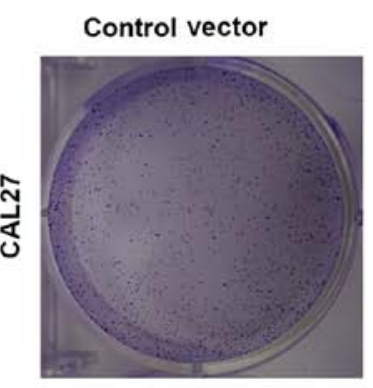

TMEM40 vector
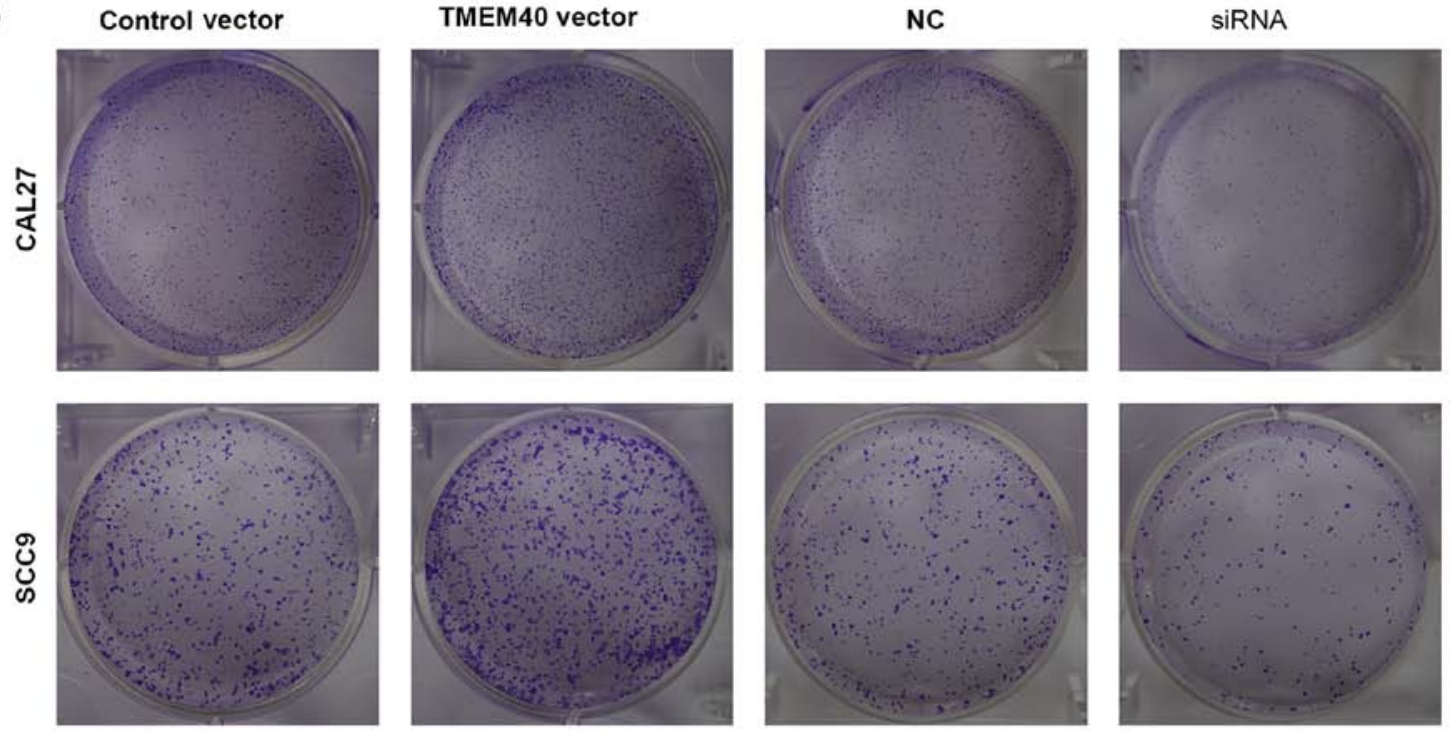

C

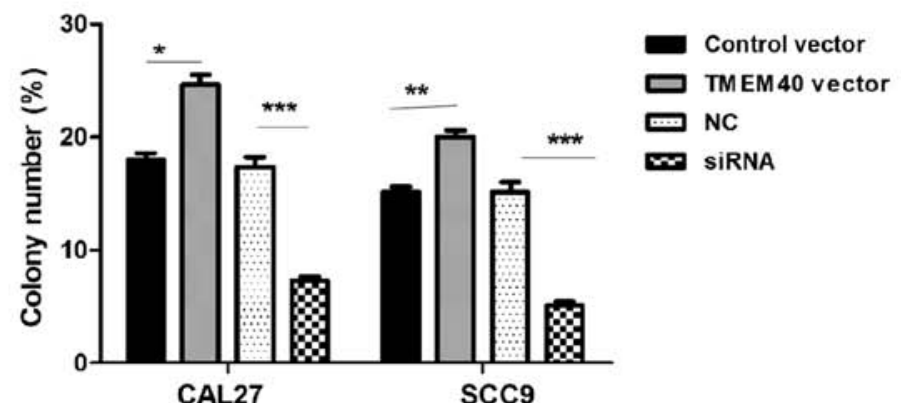

Figure 3. TMEM40 promotes cell proliferation and colony-formation abilities in TSCC cells. (A) CCK-8 results for CAL27 and SCC9 transfected with TMEM40 or control vector, or TMEM40-siRNA or negative control. (B and C) Colony formation assay results. ${ }^{*} \mathrm{P}<0.05,{ }^{* *} \mathrm{P}<0.01,{ }^{* * *} \mathrm{P}<0.001$. CCK-8, Cell Counting Kit-8; TMEM40, transmembrane protein 40; NC, negative control.

The data demonstrated that TMEM40 promoted colony formation.

Effects of TMEM40 on TSCC apoptosis and cell cycle. The influence of TMEM40 on TSCC apoptosis and cell cycle was assessed using flow cytometric assays. CAL27 and SCC9 cells were transfected with pEZ-M98-TMEM40 plasmid or empty pEZ-M98 vector and the early and late cell apoptosis ratios were observed (Fig. 4A). We calculated the total apoptosis and the results revealed that apoptosis in TMEM40-overexpressing cells was inhibited, while apoptosis in TMEM40-siRNA cells was increased (Fig. 4C; $\mathrm{P}<0.01 ; \mathrm{P}<0.001$ ). These results indicated that TMEM40 inhibited cell apoptosis in TSCC. In addition, cell cycle analysis was performed to investigate the influence of TMEM40 on TSCC cell growth (Fig. 4B). The results revealed that overexpression of TMEM40 decreased the population of the CAL27 and SCC9 cells in the G0/G1 phase and increased S-phase cells while silencing of TMEM40 increased the population of the G0/G1 phase and decreased the $\mathrm{S}$ phase (Fig. 4D; $\mathrm{P}<0.01 ; \mathrm{P}<0.001$ ). With the $\mathrm{G}_{2} / \mathrm{M}$ phase a consistent conclusion was not able to be reached. The results indicated that high expression of TMEM40 promoted proliferation and low expression of TMEM40 induced cell cycle arrest at the G0/G1 phase.

Effects of TMEM40 on cell migration and invasion. Cell migration was detected in TSCC cells using wound-healing assays. We performed a scratch wound-healing assay in 


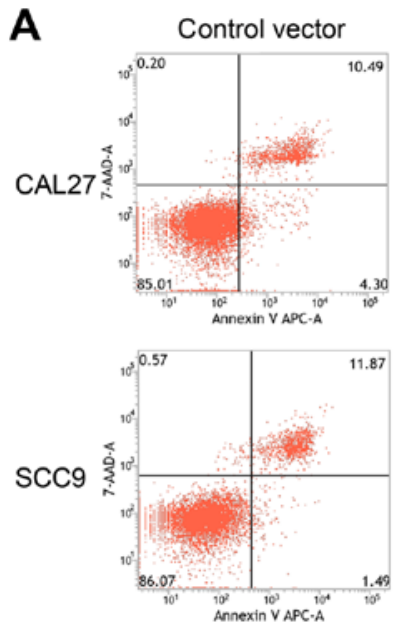

B Control vector
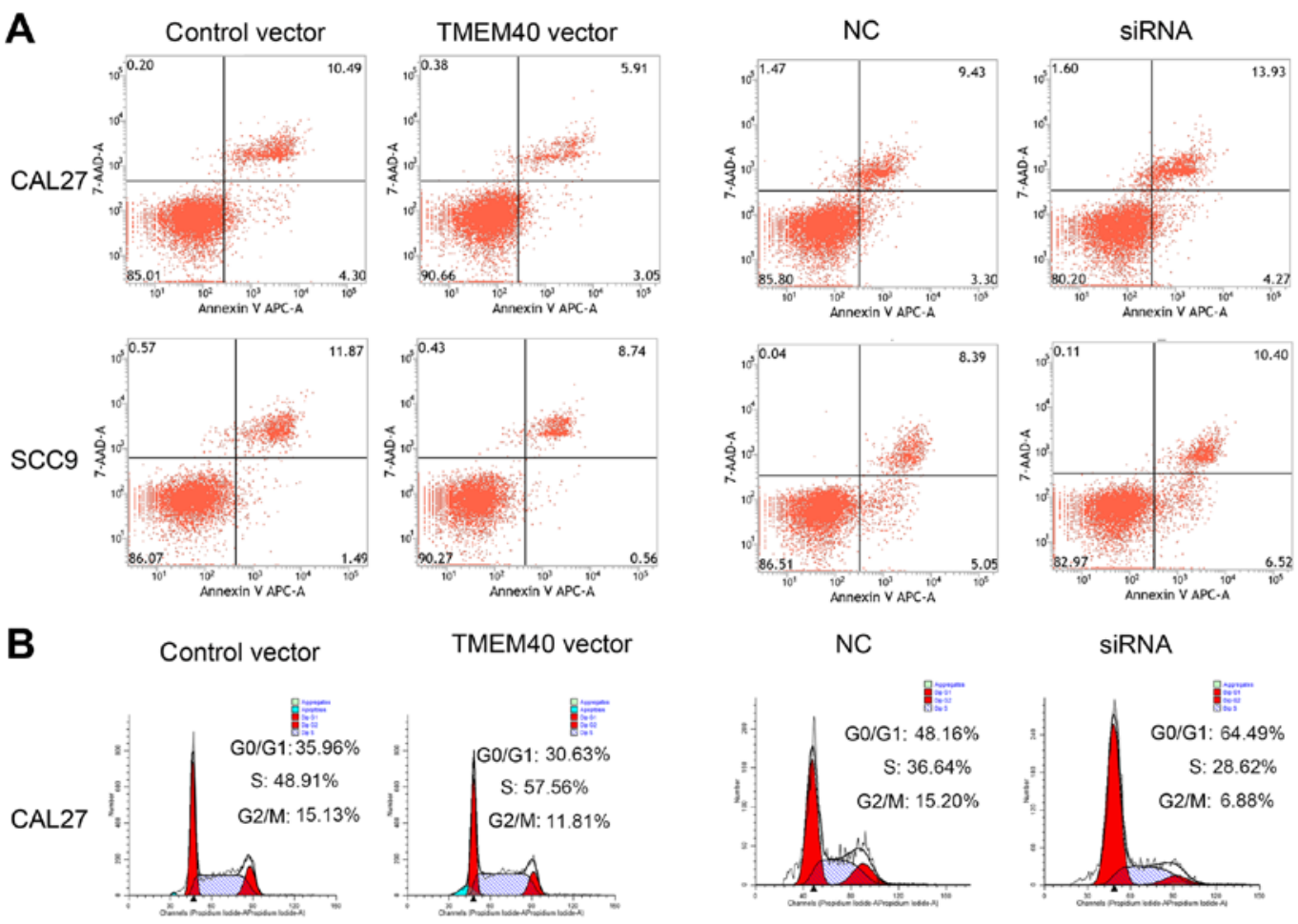

TMEM40 vector
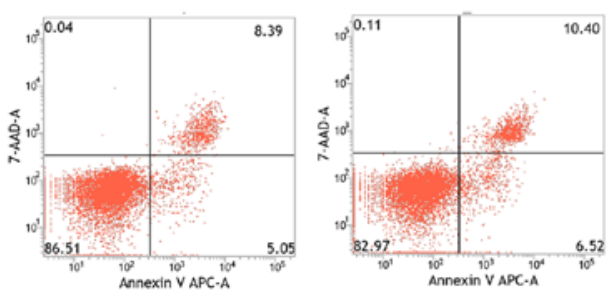

NC

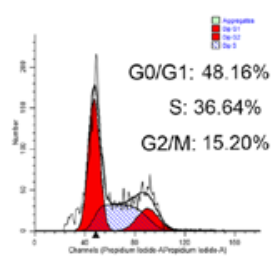

SiRNA
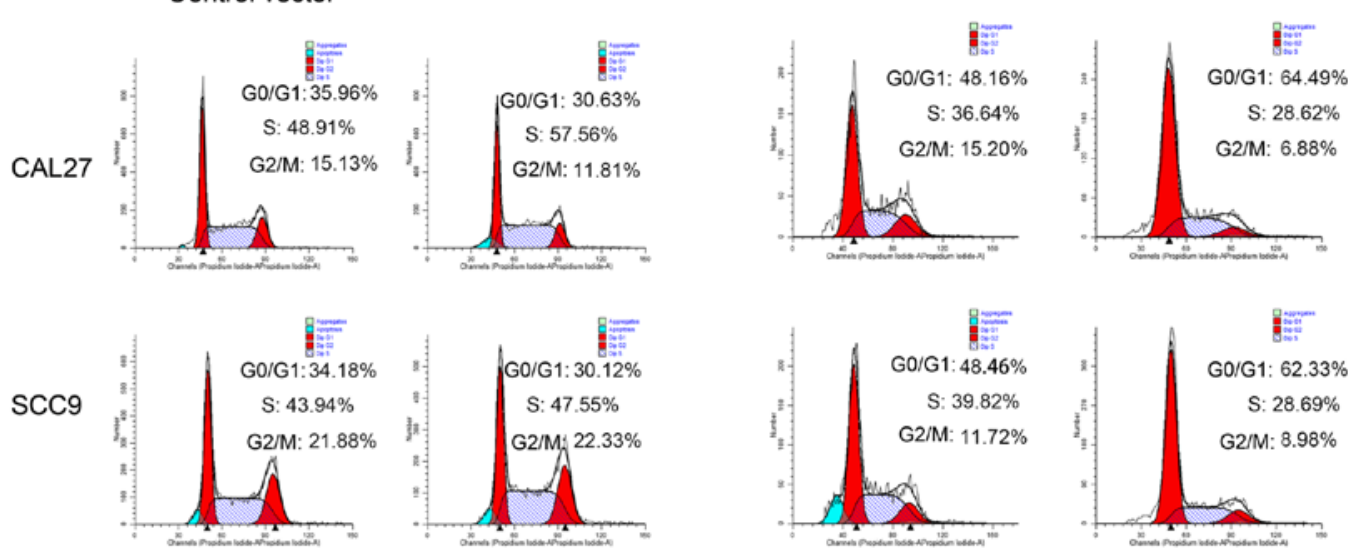

C

\section{D}
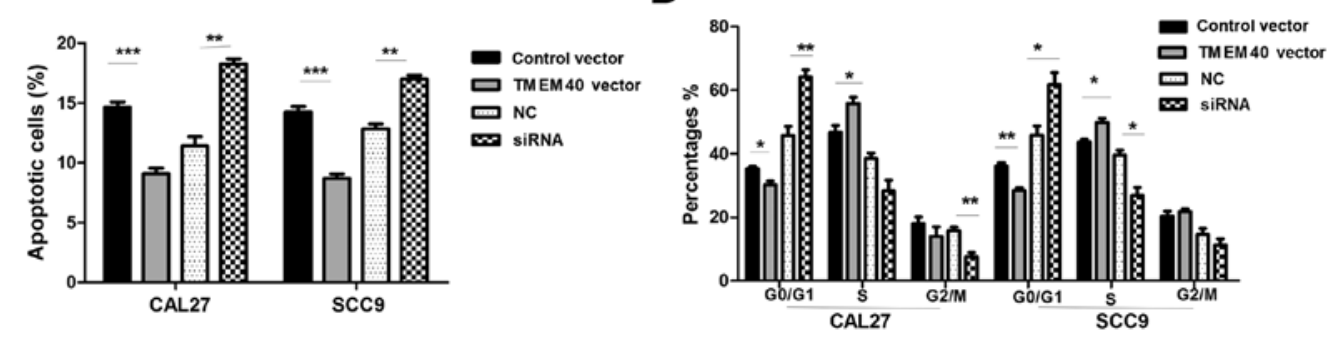

Figure 4. Effects of up- or downregulation of TMEM40 on cell apoptosis and the cell cycle in TSCC cells. CAL27 and SCC9 cells were transfected with TMEM40 or control vector, or TMEM40-siRNA or negative control. (A and C) Cell apoptosis was inhibited by overexpression of TMEM40 and increased by silencing of TMEM40. (B and D) cell cycle progression was promoted by overexpression of TMEM40 and suppressed by silencing of TMEM40. ${ }^{*}<0.05$, ${ }^{* *} \mathrm{P}<0.01,{ }^{* * *} \mathrm{P}<0.001$. TMEM40, transmembrane protein 40; TSCC, tongue squamous cell carcinoma; NC, negative control.

TMEM40-overexpressing and silenced CAL27 and SCC9 cells. As expected, cell migration activities of TSCC cells transfected with pEZ-M98-TMEM40 were significantly increased compared with the control cells transfected with empty pEZ-M98 vector. Silencing of TMEM40 significantly inhibited cell mobility compared with the control cells (Fig. 5A and $\mathrm{B} ; \mathrm{P}<0.01 ; \mathrm{P}<0.001$ ). Transwell assays were processed to determine further effects of TMEM40 on TSCC cells. The results revealed that high expression of TMEM40 significantly increased cell invasion in CAL27 and SCC9 compared with the control cells transfected with empty pEZ-M98 vector. In contrast, the inhibition of TMEM40 revealed a marked suppressive effect on cell invasion in CAL27 and SCC9 compared with the negative control (Fig. 6A and B;
$\mathrm{P}<0.01 ; \mathrm{P}<0.001)$. These findings indicated that TMEM40 promoted cell migration and invasion of TSCC.

Signaling pathway of TMEM40 in TSCC. To explore the changes on a molecular level of TMEM40 regulation of TSCC cell apoptosis and invasion, we detected alterations of tumor suppressor p53, apoptosis-associated protein Bax and invasion-associated protein MMP-9 by RT-qPCR. Knockdown of TMEM40 increased the expression levels of p53, activated Bax and decreased the levels of MMP-9 in CAL27 and SCC9 cells. These molecular changes indicated that TMEM 40 knockdown enhanced apoptosis and reduced the invasion of TSCC cells involved with p53, Bax and MMP-9 (Fig. 6C; $\mathrm{P}<0.05$; $\mathrm{P}<0.01$; $\mathrm{P}<0.001)$ 
A

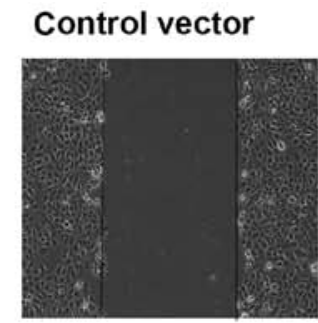

CAL27

$48 \mathrm{~h}$
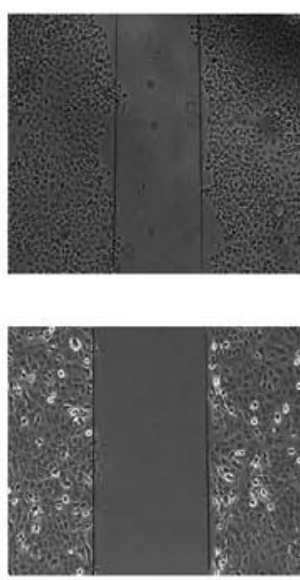

scc9

$12 \mathrm{~h}$
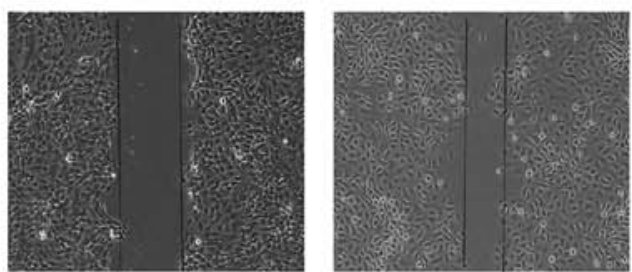

B

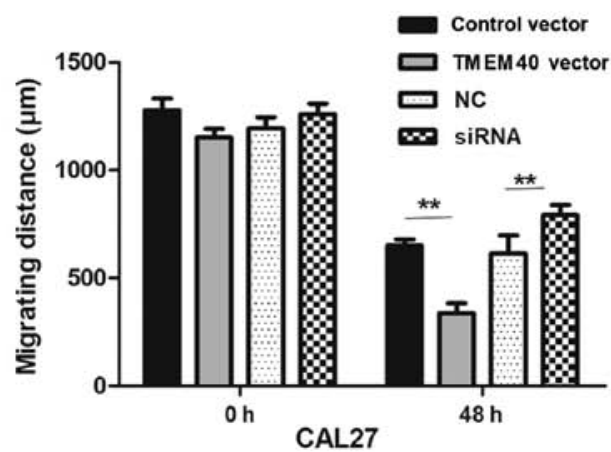

NC
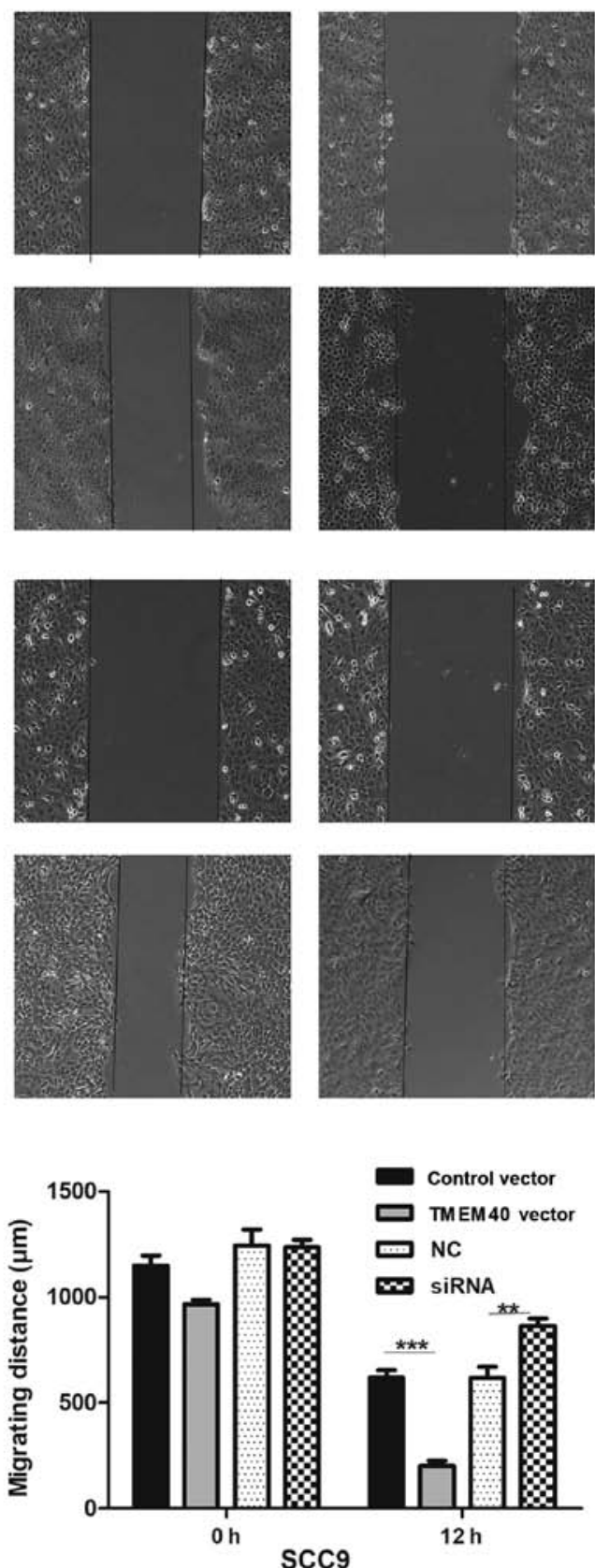

Figure 5. TMEM40 contributes to the migration of TSCC cells. (A) Cell migration abilities of CAL27 and SCC9 cells transfected with TMEM40 or control vector, or TMEM40-siRNA or negative control was assessed using a wound-healing assay. (B) Data from three independent experiments are expressed as the mean $\pm \mathrm{SD}$ (magnification, $\mathrm{x} 100$ ). ${ }^{* *} \mathrm{P}<0.01,{ }^{* * * *} \mathrm{P}<0.001$. TMEM40, transmembrane protein 40; TSCC, tongue squamous cell carcinoma; $\mathrm{NC}$, negative control.

\section{Discussion}

Squamous cell carcinoma is the most common histological type of oral cavity cancer and was found to be more aggressive than other malignancies (11). Although there are various advanced treatment options, recurrence rates are high. Local or regional relapse and cervical lymph node metastasis are threats to patients (12). Therefore, studies have been performed to elucidate the molecular mechanism of TSCC pathogenesis and explore potential biological markers of TSCC.

Genetic and epigenetic alterations associated with TSCC cause changes in proteins (13). The identification of squamous cell carcinoma-associated genes is essential for TSCC diagnosis and treatment. The genes that are critically altered in TSCC include p53 (14), epidermal growth factor receptor (15), cyclin D1 (16), retinoblastoma (17), vascular endothelial growth factor receiver (18), signal transducer and activator of transcription 3 (19), as well as other molecules. Notably, we found that TMEM40 was highly expressed in TSCC tissues compared with normal control tissues, indicating a potential role in TSCC proliferation.

The TMEM40 gene is located on chromosome 3 and alterations of chromosome 3 are closely associated with various cancers, including head and neck squamous cell carcinoma (20). Very little is known about the possible functions of TMEM40 in tumors. A previous study found that TMEM40 was highly 
A

CAL27

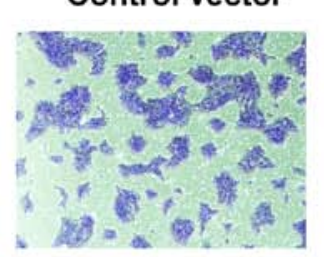

$\operatorname{scc} 9$

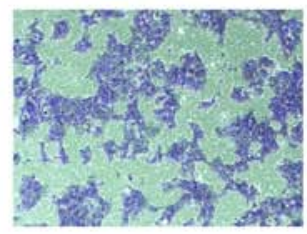

TMEM40 vector
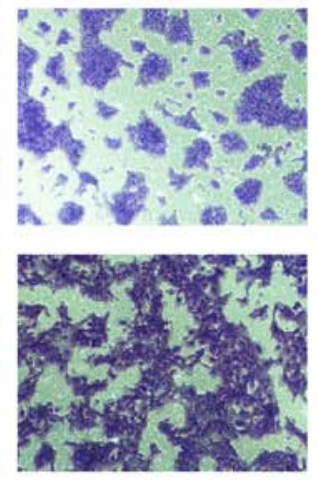

NC
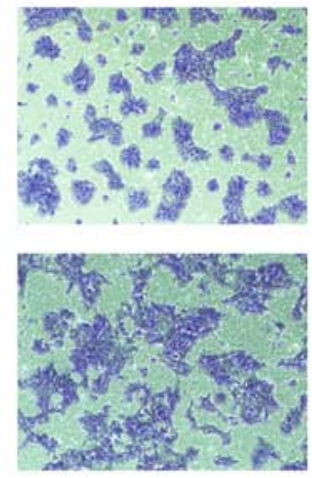

SIRNA
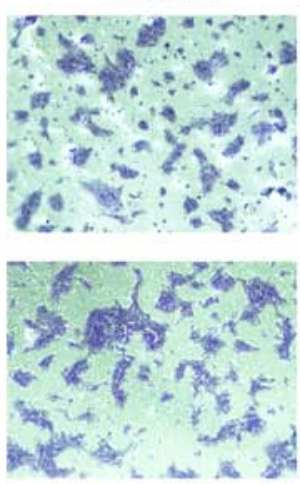

B

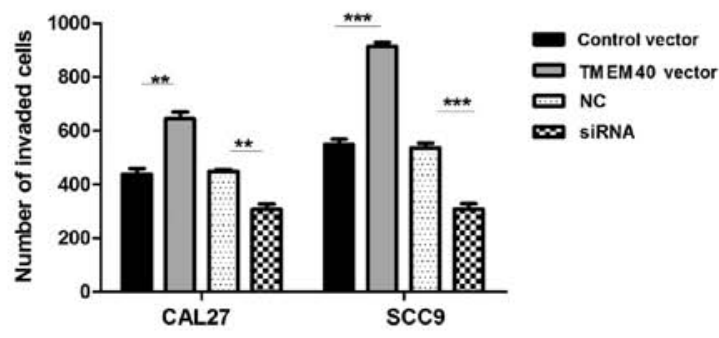

C

p53
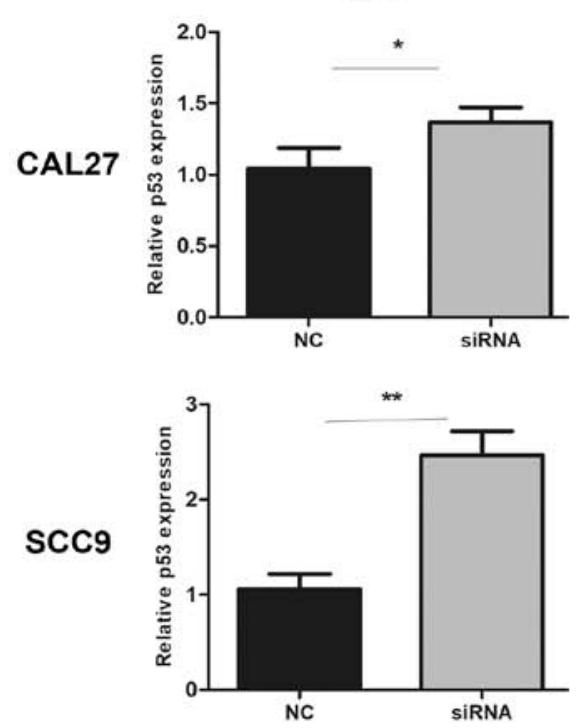

Bax
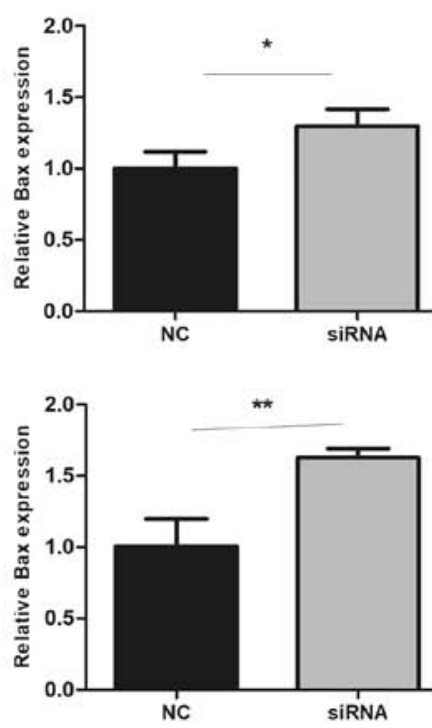

MMP9
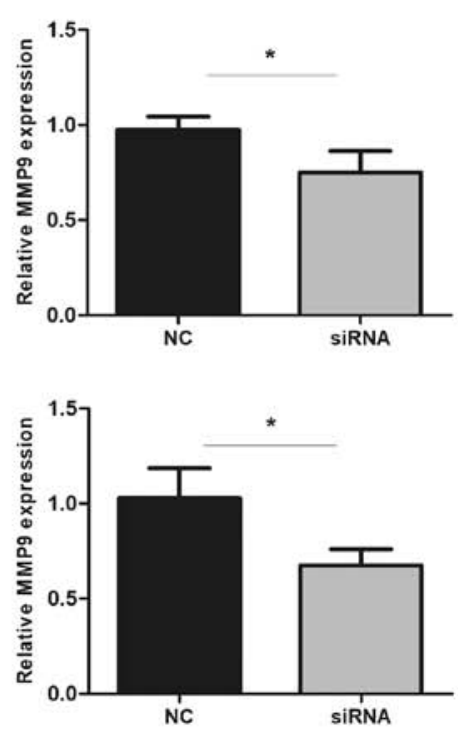

Figure 6. (A) Transwell assays were performed to examine cell invasion of CAL27 and SCC9 cells following transfection with TMEM40 or control vector, or TMEM40-siRNA or negative control. (B) Number of invaded cells compared with respective controls. (C) Knockdown of TMEM40 in CAL27 and SCC9 induced an accumulation of p53 and influenced apoptosis- and invasion-associated proteins. RT-qPCR results of p53, Bax and MMP9. Expression was normalized to GAPDH. Data are expressed as the mean $\pm \mathrm{SD}$ (magnification, $\mathrm{x} 100$ ). ${ }^{*} \mathrm{P}<0.05,{ }^{* *} \mathrm{P}<0.01,{ }^{* * * *} \mathrm{P}<0.001$. TMEM 40 , transmembrane protein 40 ; NC, negative control; RT-qPCR, reverse transcription-quantitative PCR; Bax, Bcl-2 associated X protein; MMP-9, matrix metallopeptidase 9.

expressed in bladder cancer and associated with clinicopathological parameters. In the present study, in order to explore the clinical value of TMEM40 in TSCC, a TMA was constructed, containing 50 TSCC and 10 normal tongue tissues, which were stained with TMEM40-specific dyes. And ROC analysis was employed to determine the optimal cut-off score. The results indicated that TMEM40 was frequently overexpressed in TSCC tissues and indicated that overexpression of TMEM40 may promote tumor cell growth in TSCC. To verify the functions of TMEM40, we examined the expression status of TMEM40, assessed its association with clinicopathological features in TSCC tissues and determined the biological significance in TSCC cell lines. The results implied that large tumor size, high histological grade and high clinical stage were associated with increased TMEM40 expression levels. Moreover, CCK-8, colony formation assays, wound healing assays and Transwell assays were performed to evaluate the role of TMEM40 in TSCC cells and cell apoptosis and cell cycle distribution were evaluated by flow cytometry. The results revealed that downregulation of TMEM40 expression suppressed cell proliferation, 
colony formation ability, migration and invasion and promoted apoptosis in CAL27 and SCC9 cells. In contrast, overexpression of TMEM40 increased cell proliferation, colony formation ability, migration and invasion and inhibited apoptosis.

Collectively, we found that TMEM40 expression was increased in TSCC tissues and associated with clinicopathological parameters. TMEM40 promoted proliferation, colony formation ability, cell migration and invasion in TSCC cells. These results indicated that TMEM40 contributed to the progressive features of TSCC. However, the signaling pathways of TMEM40 remain unknown and require further investigation. A previous study found that silencing of TMEM40 increased the expression levels of p53, p21, activated caspase-3, caspase- 9 and PRAP and decreased the expression levels of c-MYC and cyclin D1 in bladder cancer cells, which revealed that TMEM40 could promote bladder cancer development and progression via the p53 signaling pathway (21). The p53 tumor suppressor protein is a transcription factor that regulates several cellular stress responses, such as the control of the cell cycle, apoptosis, cell metabolism and differentiation (22). We further investigated the levels of tumor gene p53 and apoptosis-associated protein Bax by RT-qPCR. Our results revealed that knockdown of TMEM40 increased the levels of p53 and activated Bax. Bax is activated by coordinating to cytosolic p53 to initiate cell stress signaling and to induce a direct apoptotic response (23). This indicated that TMEM40 suppressed apoptosis of TSCC cells via p53- and Bax-associated pathways. Matrix metalloproteinases (MMPs) are a family of enzymes that can destroy the extracellular matrix and are connected with tumor invasion and metastasis. MMP-9 is one of most important MMPs and it has been ascertained that MMP-9 can degrade and destroy the extracellular matrix and basement membranes of tumor cells, and accelerates generation of new blood capillaries, tumor migration and invasion (24). Low levels of MMP-9 were determined in TMEM40-silenced TSCC cells indicating that MMP-9 is involved in a molecular mechanism by which TMEM40 affects cell invasion ability. In conclusion, our findings indicated that TMEM40 promoted TSCC development and progression via regulation of p53, Bax and MMP-9. However, further in-depth research will be required to verify these findings and complete the signaling pathway information.

\section{Acknowledgements}

Not applicable.

\section{Funding}

The present study was supported by the National Natural Science Foundation of China (nos. 81672588 and 81372154), the Science and Technology Planning Project of Guangdong Province, China (no. 2013B021800146) and the Natural Science Foundation of Guangdong Province, People's Republic of China (no. 2014A030313293).

\section{Availability of data and materials}

All data generated or analyzed during this study are included in this published article.

\section{Authors' contributions}

RS, YH and SL conceived, designed the study and drafted the manuscript. QZ conducted the experiments and drafted the manuscript. DH and $\mathrm{ZZ}$ carried out the statistical analysis and the acquisition of the data. YF and MF assisted with the manuscript preparation and the data analysis. MW and JZ provided technical guidance and contributed to the interpretation of the data. All authors read and approved the manuscript and agree to be accountable for all aspects of the research in ensuring that the accuracy or integrity of any part of the work are appropriately investigated and resolved.

\section{Ethics approval and consent to participate}

All the patients signed informed consent and the study was approved by the Ethics Committee of Nanfang Hospital of Southern Medical University (Guangzhou, China).

\section{Patient consent for publication}

Not applicable.

\section{Competing interests}

The authors declare that they have no conflicts of interest.

\section{References}

1. Ferlay J, Soerjomataram I, Dikshit R, Eser S, Mathers C, Rebelo M, Parkin DM, Forman D and Bray F: Cancer incidence and mortality worldwide: Sources, methods and major patterns in GLOBOCAN 2012. Int J Cancer 136: E359-E386, 2015.

2. Torre LA, Bray F, Siegel RL, Ferlay J, Lortet-Tieulent J and Jemal A: Global cancer statistics, 2012. CA Cancer J Clin 65: 87-108, 2015.

3. Hashibe M, Brennan P, Chuang SC, Boccia S, Castellsague X, Chen C, Curado MP, Dal Maso L, Daudt AW, Fabianova E, et al: Interaction between tobacco and alcohol use and the risk of head and neck cancer: Pooled analysis in the international head and neck cancer epidemiology consortium. Cancer Epidemiol Biomarkers Prev 18: 541-550, 2009.

4. Tan DS, Wang W, Leong HS, Sew PH, Lau DP, Chong FT, Krisna SS, Lim TK and Iyer NG: Tongue carcinoma infrequently harbor common actionable genetic alterations. BMC Cancer 14: 679, 2014

5. Feng Z, Xu QS, Qin LZ, Li H and Han Z: Predicting radiotherapy necessity in tongue cancer using lymph Node Yield. J Oral Maxillofac Surg 75: 1062-1070, 2017.

6. Epstein JB, Thariat J, Bensadoun RJ, Barasch A, Murphy BA, Kolnick L, Popplewell L and Maghami E: Oral complications of cancer and cancer therapy: From cancer treatment to survivorship. CA Cancer J Clin 62: 400-422, 2012.

7. Sacco AG and Cohen EE: Current treatment options for recurrent or metastatic head and neck squamous cell carcinoma. J Clin Oncol 33: 3305-3313, 2015.

8. Milanesi E, Bonvicini C, Alberici A, Pilotto A, Cattane N, Premi E, Gazzina S, Archetti S, Gasparotti R, Cancelli V, et al: Molecular signature of disease onset in Granulin mutation carriers: A gene expression analysis study. Neurobiol Aging 34: 1837-1845, 2013.

9. Zhou H, Shi R, Wei M, Zheng WL, Zhou JY and Ma WL: The expression and clinical significance of HERC4 in breast cancer. Cancer Cell Int 13: 113, 2013.

10. Livak KJ and Schmittgen TD: Analysis of relative gene expression data using real-time quantitative PCR and the $2^{-\Delta \Delta C \mathrm{~T}}$ method. Methods 25: 402-408, 2001.

11. Dissanayaka WL, Pitiyage G, Kumarasiri PV, Liyanage RL, Dias KD and Tilakaratne WM: Clinical and histopathologic parameters in survival of oral squamous cell carcinoma. Oral Surg Oral Med Oral Pathol Oral Radiol 113: 518-525, 2012. 
12. Lin CS, de Oliveira SA, Silva EL, de Matos LL, Moyses RA, Kulcsar MA, Pinto FR, Brandão LG and Cernea CR: Tumor volume as an independent predictive factor of worse survival in patients with oral cavity squamous cell carcinoma. Head Neck 39: 960-964, 2017.

13. Dumache R, Rogobete AF, Andreescu N and Puiu M: Genetic and epigenetic biomarkers of molecular alterations in oral carcinogenesis. Clin Lab 61: 1373-1381, 2015.

14. Jo DW, Kim YK and Yun PY: The influence of p53 mutation status on the anti-cancer effect of cisplatin in oral squamous cell carcinoma cell lines. J Korean Assoc Oral Maxillofac Surg 42: 337-344, 2016

15. Gao J, Ulekleiv $\mathrm{CH}$ and Halstensen TS: Epidermal growth factor (EGF) receptor-ligand based molecular staging predicts prognosis in head and neck squamous cell carcinoma partly due to deregulated EGF-induced amphiregulin expression. J Exp Clin Cancer Res 35: 151, 2016.

16. Ramos-Garcia P, Gil-Montoya JA, Scully C, Ayén A, González-Ruiz L, Navarro-Triviño FJ and González-Moles MA: An update on the implications of Cyclin D1 in oral carcinogenesis. Oral Dis 23: 897-912, 2017.

17. Murali A, Varghese BT, Kumar RR and Kannan S: Combination of genetic variants in cyclin D1 and retinoblastoma genes predict clinical outcome in oral cancer patients. Tumour Biol 37: 3609-3617, 2016

18. Li YF, Hsiao YH, Lai YH, Chen YC, Chen YJ, Chou JL, Chan MW, Lin YH, Tsou YA, Tsai MH and Tai CK: DNA methylation profiles and biomarkers of oral squamous cell carcinoma. Epigenetics 10: 229-236, 2015.
19. Gkouveris I, Nikitakis N, Karanikou M, Rassidakis G and Sklavounou A: JNK1/2 expression and modulation of STAT3 signaling in oral cancer. Oncol Lett 12: 699-706, 2016.

20. Brauswetter D, Danos K, Gurbi B, Félegyházi ÉF, Birtalan E, Meggyesházi N, Krenács T, Tamás L and Peták I: Copy number gain of PIK3CA and MET is associated with poor prognosis in head and neck squamous cell carcinoma. Virchows Arch 468: 579-587, 2016.

21. Zhang ZF, Zhang HR, Zhang QY, Lai SY, Feng YZ, Zhou Y, Zheng SR, Shi R and Zhou JY: High expression of TMEM40 is associated with the malignant behavior and tumorigenesis in bladder cancer. J Transl Med 16: 9, 2018.

22. Duplan E, Giaime E, Viotti J, Sévalle J, Corti O, Brice A, Ariga H, Qi L, Checler F and Alves da Costa C: ER-stress-associated functional link between Parkin and DJ-1 via a transcriptional cascade involving the tumor suppressor p53 and the spliced X-box binding protein XBP-1. J Cell Sci 126: 2124-2133, 2013.

23. Follis AV, Llambi F, Merritt P, Chipuk JE, Green DR and Kriwacki RW: Pin1-induced proline isomerization in cytosolic p53 mediates BAX activation and apoptosis. Mol Cell 59: 677-684, 2015.

24. Gu L, Zhang J, Shi M, Zhan Q, Shen B and Peng C: IncRNA MEG3 had anti-cancer effects to suppress pancreatic cancer activity. Biomed Pharmacother 89: 1269-1276, 2017.

This work is licensed under a Creative Commons

Attribution-NonCommercial-NoDerivatives 4.0 International (CC BY-NC-ND 4.0) License. 